REVIEWS

\title{
Motivational Interviewing Improves Medication Adherence: a Systematic Review and Meta-analysis
}

\author{
Ana Palacio, MD, MPH 1,2,3,4, Desiree Garay, $\mathrm{MD}^{7}$, Benjamin Langer ${ }^{7}$, Janielle Taylor , \\ Barbara A. Wood, MLIS , and Leonardo Tamariz, MD, MPH',2,3 \\ 'Division of Population Health and Computational Medicine, Department of Medicine, University of Miami, Miami, FL, USA; ${ }^{2}$ Department of Public \\ Health Sciences, University of Miami, Miami, FL, USA; ${ }^{3}$ Veterans Affairs Medical Center, Miami, FL, USA; ${ }^{4}$ University of Miami, Miami, FL, USA; 5 Division \\ of Health Informatics, University of Miami, Miami, FL, USA.
}

BACKGROUND: Randomized clinical trials (RCTs), mostly conducted among minority populations, have reported that motivational interviewing (MI) can improve medication adherence.

OBJECTIVES: To evaluate the impact of MI and of the MI delivery format, fidelity assessment, fidelity-based feedback, counselors' background and MI exposure time on adherence.

DATA SOURCES:We searched the MEDLINE database for studies published from 1966 until February 2015.

STUDY ELIGIBILITY CRITERIA: We included RCTs that compared MI to a control group and reported a numerical measure of medication adherence.

DATA SYNTHESIS: The main outcome was medication adherence defined as any subjective or objective measure reported as the proportion of subjects with adequate adherence or mean adherence and standard deviation. For categorical variables we calculated the relative risk (RR) of medication adherence, and for continuous variables we calculated the standardized mean difference (SMD) between the MI and control groups.

RESULTS: We included 17 RCTs. Ten targeted adherence to HAART. For studies reporting a categorical measure $(n=11)$, the pooled RR for medication adherence was higher for MI compared with control (1.17; 95 \% CI 1.05$1.31 ; \mathrm{p}<0.01)$. For studies reporting a continuous measure $(\mathrm{n}=11)$, the pooled SMD for medication adherence was positive $(0.70 ; 95 \%$ CI $0.15-1.25$; $\mathrm{p}<0.01)$ for $\mathrm{MI}$ compared with control. The characteristics that were significantly $(\mathrm{p}<0.05)$ associated with medication adherence were telephonic MI and fidelity-based feedback among studies reporting categorical measures, group MI and fidelity assessment among studies reporting continuous measures and delivery by nurses or research assistants. Effect sizes differed in magnitude, creating high heterogeneity.

CONCLUSION: MI improves medication adherence at different exposure times and counselors' educational level. However, the evaluation of MI characteristics associated with success had inconsistent results. Larger studies targeting diverse populations with a variety of chronic

Received December 5, 2014

Revised April 17, 2015

Accepted March 15, 2016

Published online May 9, 2016 conditions are needed to clarify the effect of different MI delivery modes, fidelity assessment and provision of fidelity based-feedback.

KEY WORDS: adherence; behavioral medicine; patient engagement. J Gen Intern Med 31(8):929-40 DOI: $10.1007 / \mathrm{s} 11606-016-3685-3$

(c) Society of General Internal Medicine 2016

\section{INTRODUCTION}

Lack of adherence to medications causes excess morbidity and mortality and annual health care costs of over 1 billion. ${ }^{1,2}$ In the USA, 33 to 69 percent of medication-related hospital stays were found to be due to poor adherence to medications. ${ }^{1}$ Thus, finding strategies to improve adherence to medications has become a global priority.

A promising approach is the use of cognitive-based behavior strategies such as motivational interviewing (MI). ${ }^{3,}{ }^{4} \mathrm{MI}$ is a strategy that aims to help individuals articulate and resolve their ambivalence regarding a behavior and encourages the intrinsic motivation to find individualized solutions. ${ }^{5}$ The use of MI has expanded over the years from substance abuse to adherence to HAART and more recently to adherence to other chronic medications. ${ }^{6-8}$ Most studies to date have focused on minority populations. A recent systematic review evaluated the impact of cognitive-based behavior change techniques on adherence ${ }^{3}$ and found them to be superior to usual care; however, this review included multi-layer cognitive interventions and did no focus exclusively on MI. Thus, we evaluated the impact of MI-based interventions on adherence and the effect of the MI delivery method, fidelity assessment, fidelity-based feedback to the MI counselors, MI exposure time and the educational background of the counselors. This knowledge will allow us to understand what operational factors of MI, if any, influence medication adherence.

\section{METHODS}

\section{Search Strategy}

A search was conducted through the MEDLINE database by using PubMed, which contained articles from 1966 until February 2015. We filtered all articles except those containing 
key terms such as motivational interviewing and medication adherence, compliance and persistence. More specifically, the search was performed by entering: ("medication adherence" [MeSH Terms] OR ("medication" [All Fields] AND "adherence" [All Fields]) OR "medication adherence" [All Fields]) OR ("patient compliance" [MeSH Terms] OR ("patient" [All Fields] AND "compliance" [All Fields]) OR "patient compliance" [All Fields] OR "compliance" [All Fields] OR "compliance" [MeSH Terms]) OR persistence [All Fields] AND ("motivational interviewing" [MeSH Terms] OR ("motivational" [All Fields] AND "interviewing" [All Fields]) OR "motivational interviewing" [All Fields]). All searches were conducted in February 2015 and were supplemented by manual searches of bibliographies of key relevant articles. The protocol is available for review but was not registered before data extraction started.

\section{Inclusion and Exclusion Criteria}

We included randomized trials that compared MI to one or more control groups, reported a measure of medication adherence in numerical form, had a follow-up period of any length and were published in English. We excluded studies that used MI in combination with other strategies since the isolated effect of MI could not be determined.

\section{Selection Criteria}

Two investigators (LT and BL) reviewed the abstract of each citation and identified abstracts for full-text review. Two pairs of investigators (BL and LT; AP and DG) reviewed all full-text articles and selected those that met eligibility for inclusion into the systematic review. The interrater agreement on article selection between the two pairs of investigators was $95 \%$. After completing data collection, two investigators (LT and AP) identified articles with comparable adherence measures and classified them into either categorical or continuous adherence outcomes for the purpose of the meta-analysis.

\section{Data Collection}

Two investigators completed the evidence tables (BL and DG) using previously piloted data extraction forms. LT and AP reviewed all articles in duplicate and confirmed the accuracy of the data abstracted. Differences between the two final reviewers were resolved by consensus among the four authors.

\section{Primary Outcome: Definition of Medication Adherence}

We defined two types of adherence measures: self-reported and objective. The self-reported measures included previously validated scales that defined adherence as percent or number of doses taken during a pre-specified period of time. Objective measures were collected by medication event monitoring systems (MEMS) or pharmacy claims data and defined as percent of doses taken or available during the study period or percentage of people with adequate adherence. ${ }^{1,9}$ Measures could be reported in either continuous or categorical form. Continuous measures were defined as a mean with standard deviation. Categorical measures were defined as the proportion of subjects with adequate adherence. Adequate adherence was defined as taking or having medication for 80 to $100 \%$ of the time depending on the study. ${ }^{9}$

\section{Definition of Motivational Interviewing}

We considered an intervention MI if the methods specifically described MI or the MI principles as the main strategy of counseling for that arm. ${ }^{5} \mathrm{MI}$ is a clinical method of guiding patients to make changes in the interest of their health by eliciting the patient's own motivation for change. ${ }^{5}$

\section{Definition of MI Intervention Characteristics}

MI delivery mode: We classified delivery into telephonic, in-person individual, group or mixed delivery if two or more approaches were used for the majority of participants.

Fidelity: This was considered present when the authors reported a sampling strategy for the encounters and a structured tool to measure fidelity. ${ }^{10}$

Fidelity-based feedback: This was considered present when the authors reported that feedback was given on the MI principles or skills.

Background of counselors: We classified the counselors delivering the intervention according to their level of education or background.

Exposure time: To assess the effect of time we divided the duration of the MI encounters into tertiles as there is no known cutoff to assure efficacy and calculated the RR and SMD stratified by tertiles. ${ }^{11}$

\section{Quality Evaluation}

We used the PRISMA guidelines to evaluate quality. ${ }^{12}$ For evaluation of the risk of bias at the study level we used data abstraction forms to collect: concealment of allocation, loss to follow-up, intention to treat, success of randomization, sample size calculation, missing data, recall and measurement bias, and reporting of outcomes outlined in the methods. Four investigators were responsible for completing the quality evaluation (BL, LT, DG, JT). Differences between the reviewers were resolved by consensus (lead by AP), and we calculated the interrater agreement. We evaluated risk of bias across studies by identifying selective reporting and publication bias. We used Begg's funnel plot and Egger's regression asymmetry test to evaluate publication bias. ${ }^{13}$

\section{Statistical Analysis}

We reported relevant baseline characteristics as median values with the interquartile range (IQR). To assess for heterogeneity across studies, we used the Cochran Q chi-square statistic and the I-squared statistic. We defined heterogeneity as an Isquared greater than $50 \%$. 
For the quantitative analysis we used Stata 12 (StataCorp LP, College Station, TX). Our initial analysis included all studies that reported at least one follow-up time point of medication adherence; when studies reported multiple time points we used the longest follow-up measure. Because we included studies reporting categorical or continuous medication adherence measures we stratified the analysis. Studies that reported continuous and categorical measures were included in both types of analyses. For categorical measures we calculated the relative risk (RR) of being adherent with the respective $95 \%$ confidence intervals (CIs) and p-values for all studies. Because of significant heterogeneity we used the DerSimonian and Laird random-effects model to calculate the pooled RR. For continuous measures we calculated the standardized mean difference (SMD) subtracting the mean measure of adherence in the MI intervention arm minus the mean measure of adherence in the control arm for each study. Therefore, a positive SMD indicates a higher adherence for those in the MI arm. We then calculated the pooled SMD for all studies using the DerSimonian and Laird random-effects model.

Weights for the pooled statistics were calculated using sample size for both continuous and categorical measures of adherence.

If heterogeneity was identified, we evaluated its determinants by including the suspected variables into a metaregression model. ${ }^{14}$ The metaregression model included the $\log$ of the relative risk of adequate adherence as the dependent variable and MI delivery method, fidelity assessment, fidelity-based feedback and counselors' background as the independent variables.

\section{RESULTS}

\section{Literature Search}

Our search yielded 336 abstracts. We excluded 270 studies at the abstract level and selected 66 for full-text review; of the 66 selected for full text review, we excluded 49 and included 17 studies. Figure 1 describes reasons for exclusion.

\section{Quality of the Studies and Evaluation of Bias}

Our study level bias evaluation revealed that all studies reported outcomes outlined in methods, nine studies did not report concealment of allocation, six did not report ITT analyses, nine had no sample size calculations, and eight were underpowered including six that were pilot studies. ${ }^{15-20}$

Studies that did not mention ITT $^{15,16,20-23}$ required followup visits for outcome ascertainment and used all available data for comparisons of the randomized arms. Loss to follow-up in any arm ranged from $1 \%$ to $33 \%$ (Table 1 ) with seven studies surpassing $20 \% .{ }^{16,17,18,20,22,24,25}$ One study did not report loss to follow-up by study arm, ${ }^{21}$ one had differential loss, ${ }^{16}$ and three did not comment on differences in baseline characteristics by study completion status. ${ }^{16,17,21}$ Thirteen reported no differences in characteristics by completion status, and one reported that subjects lost to follow-up in the MI group had a trend toward more depressive symptoms. ${ }^{15}$

Of studies with objective adherence measures, seven reported having missing data, ${ }^{18,}{ }^{19}, 25-29$ but only two studies had missing data in more than $20 \%$ of the sample. ${ }^{18,28}$ All described this occurrence as non-differential among study arms. All but one study ${ }^{24}$ compared baseline characteristics between arms. All had balanced groups or adjusted for minor differences. Only a pilot study by Holstad et al. ${ }^{16}$ was unable to address a difference in distribution in tribal origin that could influence the adherence measure.

The most common limitations reported by authors regarding threats to validity were: generalizability, inclusion of subjects with high adherence at baseline, ${ }^{18,20,24,25,28,30,31}$ lack of statistical power, ${ }^{15-19,28,30,32}$ and recall bias and social desirability for studies reporting only self-reported measures ${ }^{16}$, ${ }^{23}$ and including only subjects with low adherence leading to a recruitment challenge. ${ }^{17}$ The authors offered adequate explanations on how they dealt with these limitations with the exception of social desirability, which remains as a possible source of bias for those two studies.

Even though no study had differential challenges measuring adherence, many authors commented on the pitfalls of the different strategies, in particular the challenges of using MEMS. ${ }^{17,18,20,22,26,30}$

\section{Baseline Characteristics of the Studies}

Table 1 reports the baseline characteristics of the studies. The 17 studies randomized 1,376 subjects into the MI group and 1,153 subjects to the control group. Fourteen of the 17 studies were conducted in the US. Thirteen studies ${ }^{14}$ were conducted in outpatient clinics caring for the population of interest, most commonly in large metropolitan areas. Studies that used telephone-based MI exclusively identified eligible subjects from either a registry ${ }^{21}$ or from health or pharmacy benefit programs. ${ }^{27,}{ }^{32}$ The most commonly evaluated medications were highly active antiretroviral therapy (HAART). Among the 13 studies that reported race/ethnicity, 12 focused on minorities and 1963 individuals (48\%) belonged to a racial minority. The median age of the population was 43 IQR years ${ }^{33-39}$, the median percentage of women included in the studies was $65 \%$ IQR (35-86), and the median percentage of unemployed participants was $49 \%$ IQR (32-83).

\section{Description of the Motivational Interviewing Intervention}

Table 2 describes details of the intervention and control groups. Most studies included an adequate description of MI and the intervention procedures. The authors reported using known MI strategies and many described topics for specific sessions. Of the 17 retained studies, 8 delivered the MI sessions individually in person, 3 had a group MI format, 3 were 


\section{Records identified through database searching $(n=336)$}

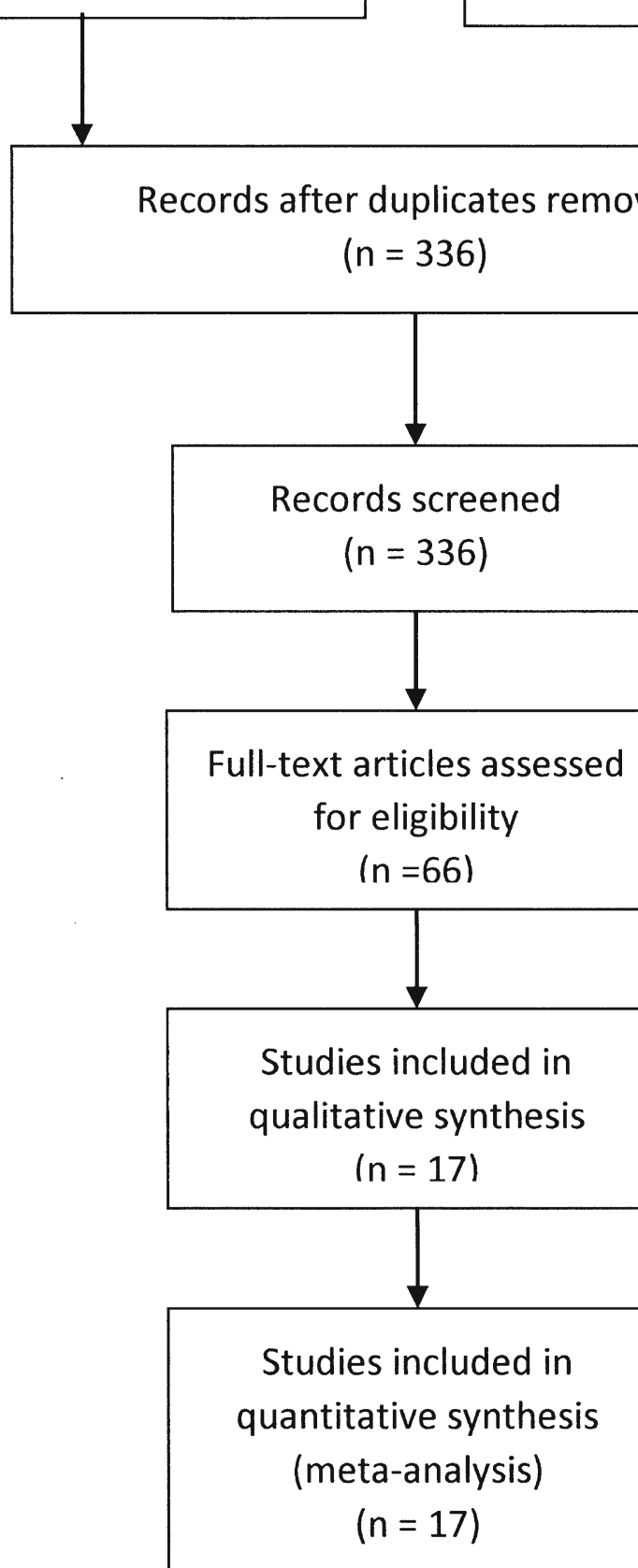

Additional records identified through other sources

$(n=0)$
Records excluded $(n=270)$
Full-text articles excluded,

$$
(n=49)
$$

Reviews $(n=20)$

Not RCT $(n=8)$

MI not intervention $(n=4)$

No adherence measure

$$
(n=14)
$$

Systematic reviews $(n=3)$

Figure 1. Flowchart of included and excluded studies

exclusively delivered over the phone, and 3 had a mixed approach.

The intervention lasted 3 months or less in eight of the studies and from 4 to 12 months in nine of them. The frequency of the MI sessions ranged from 1 week apart to 12 weeks apart for those studies with longer intervention periods. Ten studies scheduled more than one MI encounter during the first month of the study. Eleven studies reported the intended duration of each MI encounter, which ranged from $30 \mathrm{~min}$ to $2 \mathrm{~h}$ (for group MI). Most of the studies described MI encounters lasting 45 to $60 \mathrm{~min}$.

Three studies provided additional materials to the MI groups. These included video or audiotapes, educational materials, calendars or journals. 
Table 1 Baseline Characteristics of the Included Studies

\begin{tabular}{|c|c|c|c|c|c|c|}
\hline Author & Disease/medication & Sample size & Age & Females, \% & Minorities, \% & Loss to follow-up \% \\
\hline Berger $^{21}$ & Multiple sclerosis/Avonex & 212 & $45.6 \pm 9.1$ & 83 & NR & 15.6 \\
\hline Dilorio $^{20}$ & HIV/HAART & 107 & $41 \pm 6.9$ & 34 & 94 & $\begin{array}{l}\text { MI:25 } \\
\text { CG:0 }\end{array}$ \\
\hline $\operatorname{Goggin}^{2520}$ & HIV/HAART & 70 & $41 \pm 9.6$ & 29 & 79 & $\begin{array}{l}\text { MI:15 } \\
\text { CG:21 }\end{array}$ \\
\hline Golin $^{22}$ & HIV/HAART & 68 & $39.9 \pm 8.7$ & 31 & 86 & $\begin{array}{l}\text { MI:23 } \\
\text { CG:25 }\end{array}$ \\
\hline Holstad $^{26}$ & HIV/HAART & 101 & $43.5 \pm 9.1$ & 100 & 94 & $\begin{array}{l}\text { MI: } 12 \\
\text { CG: } 8\end{array}$ \\
\hline Holstad $^{16}$ & HIV/HAART & 28 & $31 \pm 5.3$ & 100 & 100 & $\begin{array}{l}\text { MI: } 6.7 \\
\text { CG:33 }\end{array}$ \\
\hline Ingersoll $^{17}$ & HIV/HAART & 19 & $44 \pm 5.1$ & 63 & 94.8 & $\begin{array}{l}\text { MI: } 25 \\
\text { CG:18 }\end{array}$ \\
\hline Interian $^{19}$ & Depression/antidepressants & 26 & $40.2 \pm 10.8$ & 73 & 100 & $\begin{array}{l}\text { MI:12 } \\
\text { CG:12 }\end{array}$ \\
\hline Konkle-Parker ${ }^{15}$ & HIV/HAART & 33 & $34.9 \pm 7.4$ & 36 & 82 & $\begin{array}{l}\text { MI:15 } \\
\text { CG:17 }\end{array}$ \\
\hline Lavoie $^{18}$ & Asthma /ICS & 26 & $52 \pm 15.0$ & 50 & NR & $\begin{array}{l}\text { MI:27 } \\
\text { CG:21 }\end{array}$ \\
\hline Ogedegbe ${ }^{29}$ & Hypertension/antihypertensives & 95 & $53.4 \pm 11.3$ & 50 & 100 & $\begin{array}{l}\text { MI:3 } \\
\text { CG: } 10\end{array}$ \\
\hline Palacio $^{27}$ & Cardiovascular/antiplatelet & 227 & $69.1 \pm 8.6$ & 44 & 100 & $\begin{array}{l}\text { MI:6 } \\
\text { CG:7 }\end{array}$ \\
\hline Parsons $^{24}$ & HIV/HAART & 65 & $43.6 \pm 6.9$ & 21 & 94 & $\begin{array}{l}\text { MI:18 } \\
\text { CG:13 }\end{array}$ \\
\hline Pradier $^{23}$ & HIVHAART & 123 & $40[35-49]$ & 29 & NR & $\begin{array}{l}\text { MI }:<1 \\
\text { CG }:<1\end{array}$ \\
\hline Samet $^{28}$ & HIV/HAART & 74 & $42.5 \pm 7.9$ & 22 & 66 & $\begin{array}{l}\text { MI:4 } \\
\text { CG:9 }\end{array}$ \\
\hline Solomon $^{32}$ & Osteoporosis & 1050 & $77.8 \pm 6.4$ & 94 & 10 & $\begin{array}{l}\text { MI:9 } \\
\text { CG: } 1.5\end{array}$ \\
\hline Zwikker $^{31}$ & Rheumatoid arthritis/DMD & 63 & $60.4 \pm 12.1$ & 67 & NR & $\begin{array}{l}\text { MI:16 } \\
\text { CG:2 }\end{array}$ \\
\hline
\end{tabular}

NR: not reported

ICS: Inhaled corticosteroids

DMD: Disease-modifying drugs

MI: Motivational interviewing

CG: Control group

\section{Description of Medication Adherence}

Table 2 reports the tools used to measure adherence, the definition of adherence used, the measurement time points for each study and whether the measure was reported in categorical or continuous format. Medication adherence ascertainment and reporting were heterogeneous across studies. Of the 17 studies, 7 used only an objective adherence measure, 6 used only self-reported measures, and 4 reported both types of measures. Of the 11 studies that had an objective measure of adherence, 6 used MEMS while 5 used pharmacy claims data to capture adherence. The self-reported measures varied more and included a number of validated tools to estimate mean adherence or percent of subjects with adequate adherence over a previous set of days that ranged from 4 to 30 days. Eleven studies reported the adherence measures in categorical form and 11 in continuous form, and 5 reported both types. The median follow-up for the evaluation of adherence was 6 months IQR. ${ }^{4-10,12,13}$

\section{Quality of Motivational Interviewing}

Table 3 summarizes the training, fidelity assessment and fidelity-based feedback reported by each study. These are considered key quality metrics for MI. ${ }^{5}$ Ten studies reported the amount of time dedicated to MI training at baseline, which ranged from 4 to $40 \mathrm{~h}$. Most studies reported approximately $24 \mathrm{~h}$ of training. Regarding MI fidelity assessment, 9 of the 17 studies used a validated assessment tool among a random sample of encounters, and 3 described a similar process without mentioning a specific tool. Ten of these studies reported providing fidelity-based feedback and booster MI trainings.

Five studies described quality assurance methods that did not directly assess the fidelity of the MI.

\section{Meta-analysis results}

Publication Bias. We did not detect publication bias using Begg's funnel plot, and the beta-coefficient of the regression asymmetry was not significant (-0.009 with a p-value of 0.81$)$.

\section{Impact of MI on Medication Adherence}

For studies reporting the proportion of patients with adequate adherence $(n=11)$ the pooled RR of being adherent for MI was $1.17(95 \%$ CI $1.05-1.31 ; \mathrm{p}<0.01)$ when compared to the control group.

For studies reporting a continuous measure $(n=11)$, the SMD was 0.70 (95\% CI $0.15-1.25 ; \mathrm{p}<0.01)$ for MI when compared to the control group. 
Table 2 Description of the Motivational Interviewing Characteristics and Adherence Measures

\begin{tabular}{|c|c|c|c|c|c|}
\hline Author & $\begin{array}{l}\text { MI delivery } \\
\text { mode }\end{array}$ & $\begin{array}{l}\text { Definition of } \\
\text { adherence/ evalua- } \\
\text { tion time points/ var- } \\
\text { iable type }\end{array}$ & $\begin{array}{l}\text { MI description and exposure } \\
\text { time to MI }\end{array}$ & Control description & Interventionist \\
\hline Berger $^{21}$ & $\begin{array}{l}\text { Telephone } \\
\text { counseling }\end{array}$ & $\begin{array}{l}\text { SR } \\
\text { Discontinuation of } \\
\text { treatment at } 3 \text { months } \\
\text { Continuous }\end{array}$ & $\begin{array}{l}\text { Software-guided MI calls } \\
\text { every } 2 \text { to } 4 \text { weeks for } 3 \\
\text { months depending on stage of } \\
\text { readiness. MI exposure not } \\
\text { specified }\end{array}$ & $\begin{array}{l}\text { Access to call center via toll- } \\
\text { free hotline }\end{array}$ & $\begin{array}{l}\text { Three members of the } \\
\text { call center }\end{array}$ \\
\hline Dilorio $^{20}$ & $\begin{array}{l}\text { Individual } \\
\text { counseling } \\
\text { and telephone } \\
\text { as needed }\end{array}$ & $\begin{array}{l}\text { MEMS } \\
\% \text { Doses taken } \\
\% \text { Doses taken on } \\
\text { time } \\
\text { At } 3,6 \text { and } 12 \text { months } \\
\text { Continuous }\end{array}$ & $\begin{array}{l}\text { Five MI sessions over } 3 \\
\text { months. Eighty percent held in } \\
\text { person and lasted on average } \\
\text { between } 20-90 \text { min. Telephone } \\
\text { sessions (for sessions } 2-5 \text { ) } \\
\text { were conducted as needed for } \\
\text { participants who were unable } \\
\text { to meet the counselor in the } \\
\text { clinic. Minimum exposure } \\
\text { time: } 170 \text { min (median) }\end{array}$ & $\begin{array}{l}\text { Usual adherence education } \\
\text { provided at the clinic }\end{array}$ & $\begin{array}{l}\text { Study nurses trained in } \\
\text { MI }\end{array}$ \\
\hline Goggin $^{25}$ & $\begin{array}{l}\text { Individual and } \\
\text { telephone calls }\end{array}$ & $\begin{array}{l}\text { EDM } \\
-\% \text { Doses taken } \\
-\% \text { Doses taken on } \\
\text { time } \\
\text { At } 3,6,9 \text { and } \\
12 \text { months } \\
\text { Continuous }\end{array}$ & $\begin{array}{l}\text { Six MI counseling sessions } \\
\text { (weeks } 0,1,2,6,11 \text { and } 23 \text { ) } \\
\text { and } 4 \text { telephone sessions } \\
\text { (weeks } 4,9,15 \text { and } 19 \text { ). On } \\
\text { average, sessions lasted } 25 \\
\text { min. Average exposure } 150 \\
\text { min }\end{array}$ & $\begin{array}{l}\text { Medical care and counseling } \\
\text { as usual from their clinic } \\
\text { providers }\end{array}$ & $\begin{array}{l}\text { Master's degree level } \\
\text { trained by a licensed } \\
\text { clinical psychologist }\end{array}$ \\
\hline Golin $^{22}$ & $\begin{array}{l}\text { Individual } \\
\text { counseling }\end{array}$ & $\begin{array}{l}\text { CAS: pill count, SR } \\
\text { EDM: } \\
>95 \% \text { adherent } \\
\text {-mean adherence } \\
-\% \text { Doses taken } \\
\text { At } 3 \text { months } \\
\text { Continuous and } \\
\text { categorical }\end{array}$ & $\begin{array}{l}\text { During } 12 \text { weeks subjects } \\
\text { received a } 20 \text {-min audiotape } \\
\text { and booklet, two MI sessions } \\
\text { at weeks } 4 \text { and } 8 \text {, and a } \\
\text { mailing } 2 \text { weeks after each } \\
\text { session. Exposure time: not } \\
\text { reported }\end{array}$ & $\begin{array}{l}\text { Similar format but providing } \\
\text { only general HIV information } \\
\text { without using MI techniques }\end{array}$ & $\begin{array}{l}\text { Master's degree level } \\
\text { health educator }\end{array}$ \\
\hline Holstad $^{16}$ & $\begin{array}{l}\text { Group } \\
\text { counseling }\end{array}$ & $\begin{array}{l}\text { MEMS Track-caps: } \\
-\% \text { Doses taken } \\
-\% \text { Doses taken on } \\
\text { schedule } \\
\text { At } 3 \text { and } 6 \text { months } \\
\text { Categorical }\end{array}$ & $\begin{array}{l}\text { Eight } 1.5-2 \text { h group MI } \\
\text { sessions during } 9 \text { months. } \\
\text { MI Exposure time: } 720-960 \\
\text { min }\end{array}$ & $\begin{array}{l}1.5-2-\mathrm{h} \text { sessions on nutrition, } \\
\text { exercise, stress reduction and } \\
\text { women's health }\end{array}$ & Trained MI nurses \\
\hline Holstad $^{26}$ & $\begin{array}{l}\text { Group } \\
\text { counseling }\end{array}$ & $\begin{array}{l}\text { SR: AGAS, VAS and } \\
\text { ACTG scales: } \\
\text { Mean adherence } \\
\text {-proportion of doses } \\
\text { taken in prior } 30 \text { days } \\
\text {-never missing in } \\
30 \text { days } \\
\text { At } 6 \text { months } \\
\text { Continuous and } \\
\text { categorical }\end{array}$ & $\begin{array}{l}\text { Eight } 1.5-2 \text {-h MI group } \\
\text { sessions during 6-month MI } \\
\text { exposure: } 720-960 \mathrm{~min}\end{array}$ & $\begin{array}{l}\text { Eight } 1.5-2-\mathrm{h} \text { health } \\
\text { education sessions focusing } \\
\text { on nutrition, exercise, stress } \\
\text { reduction and women's } \\
\text { health }\end{array}$ & Health workers \\
\hline Ingersoll $^{17}$ & $\begin{array}{l}\text { Individual } \\
\text { counseling }\end{array}$ & $\begin{array}{l}\text { SR: 14-day TLFB: } \\
\% \text { Pills taken per day } \\
\text { At } 3 \text { and } 6 \text { months } \\
\text { Continuous }\end{array}$ & $\begin{array}{l}6 \text { MI sessions plus feedback } \\
\text { and skills building over } 8 \\
\text { weeks. Each session lasted } \\
\text { between } 45 \text { and } 60 \text { min. MI } \\
\text { exposure } 270-360 \text { min }\end{array}$ & $\begin{array}{l}\text { Equivalent time to offer } \\
\text { information about HAART } \\
\text { and crack cocaine use }\end{array}$ & $\begin{array}{l}\text { Master's or doctoral } \\
\text { degree in clinical } \\
\text { psychology, social } \\
\text { work or counseling }\end{array}$ \\
\hline Interian $^{19}$ & $\begin{array}{l}\text { Individual } \\
\text { counseling }\end{array}$ & $\begin{array}{l}\text { MEMS Cap and } \\
\text { Medication Taking } \\
\text { Scale: } \\
\% \text { Doses taken } \\
\text { At } 5 \text { weeks and } \\
5 \text { months } \\
\text { Continuous }\end{array}$ & $\begin{array}{l}\text { Three } 60 \text {-min MI sessions } \\
\text { during } 5 \text { months. } \\
\text { MI exposure: } 180 \mathrm{~min}\end{array}$ & $\begin{array}{l}\text { Pharmacotherapy, as well as } \\
\text { some psychotherapy } \\
\text { treatment }\end{array}$ & $\begin{array}{l}\text { Clinical psychologist } \\
\text { and psychology } \\
\text { doctoral students }\end{array}$ \\
\hline $\begin{array}{l}\text { Konkle- } \\
\text { Parker }^{15}\end{array}$ & $\begin{array}{l}\text { Individual } \\
\text { counseling and } \\
\text { telephone calls }\end{array}$ & $\begin{array}{l}\text { SR: } 3-\text { to } 4 \text { - week } \\
\text { adherence recall by } \\
\text { VAS } \\
\text { Pharmacy refill rate } \\
>90 \% \text { adherent } \\
\text { At } 26 \text { weeks } \\
\text { Continuous }\end{array}$ & $\begin{array}{l}\text { Two } 30-60 \text { min sessions with } \\
\text { the PI at weeks } 1 \text { and } 2 \text { and } \\
\text { then six telephone call sessions } \\
\text { tapering during } 24 \text { weeks. } \\
\text { Telephone sessions averaged } \\
\text { less than } 10 \text { min. MI exposure: } \\
120 \text { min }\end{array}$ & Usual care & $\begin{array}{l}\text { PI and a nurse } \\
\text { practitioner trained in } \\
\text { MI }\end{array}$ \\
\hline Lavoie $^{18}$ & Individual & $\begin{array}{l}\% \text { Pharmacy refills } \\
\text { Mean change in } \\
\text { percent of refills } \\
\text { At } 6 \text { and } 12 \text { months } \\
\text { Continuous }\end{array}$ & $\begin{array}{l}\text { Three to five individual } 15-30- \\
\text { min sessions over a } 4-6 \text {-week } \\
\text { period. The educator and } \\
\text { patient decided together if } \\
\text { more than } 3 \text { sessions were } \\
\text { needed. MI exposure time: } 60 \\
\text { to } 150 \mathrm{~min}\end{array}$ & Standard of care & $\begin{array}{l}\text { Psychologist trained in } \\
\text { motivational } \\
\text { interviewing }\end{array}$ \\
\hline
\end{tabular}


Table 2. (continued)

\begin{tabular}{|c|c|c|c|c|c|}
\hline Author & $\begin{array}{l}\text { MI delivery } \\
\text { mode }\end{array}$ & $\begin{array}{l}\text { Definition of } \\
\text { adherence/ evalua- } \\
\text { tion time points/ var- } \\
\text { iable type }\end{array}$ & $\begin{array}{l}\text { MI description and exposure } \\
\text { time to MI }\end{array}$ & Control description & Interventionist \\
\hline Ogedege $^{29}$ & $\begin{array}{l}\text { Individual } \\
\text { counseling }\end{array}$ & $\begin{array}{l}\text { MEMS pill caps } \\
\% \text { Adherent at } \\
12 \text { months } \\
\text { Categorical }\end{array}$ & $\begin{array}{l}30-40 \text {-min MI sessions at } 3,6 \text {, } \\
9 \text { and } 12 \text { month } \\
\text { MI exposure time: } 120 \mathrm{~min}\end{array}$ & Usual care & Trained RAs \\
\hline Palacio $^{27}$ & Telephone & $\begin{array}{l}\text { Pharmacy claims data } \\
\text {-MPR } \\
\text {-MPR } \geq 80 \% \\
\text { Self report: MMAS-4 } \\
\text { At } 12 \text { months } \\
\text { Continuous and } \\
\text { categorical }\end{array}$ & $\begin{array}{l}\text { Four } 20 \text { - } 30 \text {-min sessions } \\
\text { during a } 12 \text {-month period } \\
\text { (calls every } 3 \text { months) } \\
\text { MI exposure: } 80 \text { to } 120 \mathrm{~min}\end{array}$ & Mailed educational DVD & MI-trained nurses \\
\hline Parsons $^{24}$ & Individual & $\begin{array}{l}\text { SR: 14-day TLFB } \\
\text { \% Doses taken } \\
\% \text { Days with perfect } \\
\text { adherence } \\
\text { At } 3 \text { and } 6 \text { months } \\
\text { Categorical }\end{array}$ & $\begin{array}{l}\text { Eight } 60 \text {-min sessions over a } \\
\text { period of } 12 \text { weeks } \\
\text { The first session delivered } \\
\text { immediately on completion of } \\
\text { the baseline assessment. } \\
\text { MI exposure time: } 480 \text { min }\end{array}$ & $\begin{array}{l}8 \text { Sessions to give } \\
\text { information on HIV, HAART } \\
\text { and alcohol }\end{array}$ & $\begin{array}{l}\text { Master's degree } \\
\text { prepared counselors }\end{array}$ \\
\hline Pradier $^{23}$ & $\begin{array}{l}\text { Individual } \\
\text { counseling }\end{array}$ & $\begin{array}{l}\text { SR: 4-day recall } \\
\% \text { Adherent at } \\
6 \text { months } \\
\text { Categorical }\end{array}$ & $\begin{array}{l}\text { Three 45-60-min MI sessions } \\
\text { at months } 0,2 \text { and } 4 \text {. } \\
\text { MI exposure time } 135-180 \mathrm{~min}\end{array}$ & $\begin{array}{l}\text { Clinical follow-up every } 2-3 \\
\text { months }\end{array}$ & Trained nurses \\
\hline Samet $^{28}$ & $\begin{array}{l}\text { Individual } \\
\text { counseling }\end{array}$ & $\begin{array}{l}\text { SR: } 3 \text { - and 30-day } \\
\text { ACTG scale } \\
\text {-mean 30-day adher- } \\
\text { ence } \\
-95 \% \text { adherent in last } \\
30 \text { days } \\
\text { At } 2-6 \text { and } 12-13 \\
\text { months } \\
\text { Categorical }\end{array}$ & $\begin{array}{l}\text { Initial } 60 \text {-min individual, a } \\
\text { follow-up home visit within } \\
\text { the first } 3 \text { weeks, and } 2 \text { subse- } \\
\text { quent } 15-30 \text {-min appointments } \\
\text { at } 1 \text { month and } 3 \text { months. } \\
\text { MI exposure time: } 90-120 \text { min }\end{array}$ & $\begin{array}{l}\text { Standard care for HIV } \\
\text { infection, included verbal and } \\
\text { written instructions about } \\
\text { optimal medication }\end{array}$ & Nurse trained in $\mathrm{MI}$ \\
\hline Solomon $^{32}$ & $\begin{array}{l}\text { Telephone } \\
\text { counseling }\end{array}$ & $\begin{array}{l}\text { Median MPR } \\
\text { MPR }>80 \% \\
\text { At } 12 \text { months } \\
\text { Continuous and } \\
\text { categorical }\end{array}$ & $\begin{array}{l}\text { Ten phone-based MI sessions } \\
\text { during } 12 \text { months } \\
\text { MI exposure: not specified }\end{array}$ & Mailed educational materials & Health educators \\
\hline Zwikker $^{31}$ & $\begin{array}{l}\text { Group } \\
\text { counseling }\end{array}$ & $\begin{array}{l}\text { SR: CQR and MARS } \\
\text { MPR } \\
\% \text { Adherent } \\
\text { At } 6 \text { and } 12 \text { months } \\
\text { Categorical }\end{array}$ & $\begin{array}{l}\text { Intervention consisted of two } \\
\text { MI-guided group sessions (1 } \\
\text { week apart) }\end{array}$ & $\begin{array}{l}\text { Brochures about DMARDS } \\
\text { they were using }\end{array}$ & Pharmacists \\
\hline
\end{tabular}

SR: Self report:

MEMS: Medication Event Monitoring System

CAS: Composite adherence score

EDM: Electronic drug monitor

AGAS: Anti-retroviral medication General Adherence Scale

VAS: visual analog scale (VAS)

$A C T G$ : adherence questionnaire

14-TLFB: 14-day timeline follow-back

MMAS-4: 4-item Morisky Medication Adherence Scale

MPR: Medication possession ratio

CQR: Compliance Questionnaire Rheumatology

MARS: Medication Adherence Report Scale

DMARDS: disease modifying anti-rheumatic drugs

There was statistical heterogeneity (I-squared $>50 \%$ and $\mathrm{p}<0.01)$ for both types of adherence measures. The main reason for the heterogeneity was the MI delivery mode as reflected by its beta coefficient in the metaregression model $(0.11 ; 95 \%$ CI $0.06-0.17, \mathrm{p}<0.01)$.

\section{The Effect of MI Intervention Characteristics on Adherence}

Table 4 summarizes the results of the subgroup analyses. Individual and mixed MIs were not superior to the control intervention at improving adherence. Phone-based MI improved adherence measured categorically and group MI improved adherence measured continuously. These latter analyses had small between-studies variance in effect size compared to telephonic continuous and group categorical measures that had larger differences, although with the same directionality (Fig. 2a and b).

MI was superior to the control group among studies that used a fidelity assessment tool and reported continuous adherence measures. Providing fidelity-based feedback was superior among studies reporting categorical measures. 
Table 3 Description of MI Training, Fidelity Assessment and Fidelity-Based Feedback

\begin{tabular}{llll}
\hline \hline Author & MI training & Fidelity assessment & Fidelity-based feedback \\
\hline Berger $^{21}$ & $\begin{array}{l}\text { 8-h baseline training including use of } \\
\text { software and case scenarios }\end{array}$ & No & No
\end{tabular}

Dilorio $^{20} \quad$ Investigators who are psychologists provided $24 \mathrm{~h}$ of in-class presentation of MI theory and methods and practice sessions. Following these sessions, the nurses' skills were tested using a standardized patient approach

Goggin $^{25} \quad$ Counselors were master's level professionals trained and supervised by a licensed clinical psychologist.

Golin $^{22} \quad$ Counselors received $24 \mathrm{~h}$ of training over a 2-month period, conducted by members of the MI Network of Trainers. A standardized MI guide composed of 7 main steps was used

Holstad $^{16} \quad 24 \mathrm{~h}$ of training during a 1-week period and booster MI training throughout the study. Included MI theory and practice, HIV and ART information and group management skills. The MI training included role play and two videotaped interviews with standardized patients

Holstad $^{26}$ Same as above but without the two videotaped sessions

Ingersoll $^{17}$ The supervisors and principal investigator, all members of the motivational Interviewing Network of Trainers, facilitated the training and supervision of therapists. Therapists also attended external MI workshops and trainings

Interian $^{19} \quad$ Not mentioned

Konkle- The interventionist was trained in MI at

Parker $^{15}$ baseline

Lavoie $^{18} \quad$ Psychologist who delivered the MI intervention received 2-day intensive MI workshop training from an accredited MI Network Trainer

Ogedegbe $^{29} \quad$ Experienced MI trainers conducted two 8-h training sessions the first year and a 1-day booster session yearly thereafter. It included lectures and role-plays

Palacio $^{27}$ 3-Day workshop conducted by an experienced MI trainer and by a specialist in the MITI Coding system. Nurses also received 1-week training on coronary artery disease, antiplatelet medications, predictors of adherence and study-specific protocols

Parsons $^{24} \quad$ Counselors completed significant training in MI

Pradier $^{23} \quad$ Psychologists who were part of the research team conducted a 5-day intensive MI training course

Samet $^{28} \quad$ The nurse had prior HIV and substance abuse expertise and was specifically trained in motivational interviewing to address substance abuse and HIV medication adherence

Solomon $^{32} \quad$ Half-day training at baseline, including role-playing and lectures. MI reinforced via 1-2 teleconferences a month with a behavioral scientist
Yes. A psychologist and graduate students trained in MI counseling evaluated MI tapes and coded them for fidelity using a structure coding form. These were used to assess MI nurses adherence to MI script and MI skills Yes. All sessions were recorded and a random sample was coded for fidelity using a 26-item coding scheme with a 7point scale ranging from poor to excellent No. Not by an independent rater. At the end of each MI session, counselors recorded the content of the 7 protocol steps on an MI data recording sheet

Yes. All MI and control group sessions were videotaped and were reviewed by a therapist trained in MI. This rater used a structured coding form to evaluate the fidelity of the intervention in a sample of $20 \%$ of the group sessions

No. Not by independent rater. Study subjects evaluated each session for the use of MI

Yes, but unknown tool. All sessions were audio- or videotaped, and these were used in weekly group and individual supervision.

Fidelity was assessed during weekly supervision of therapists conducting the interventions via review of videotaped sessions

Yes. Fidelity was assessed with the MITI Scale. An independent rater scored each of the first two sessions for each META participant

Yes. An MI expert coded a random $10 \%$ sample of the audiotapes using the MITI 2.0 Scale

Yes. All intervention sessions were audiotaped and a random $10 \%$ were evaluated by an independent MI expert using the Motivational Interviewing Skill Code

Yes, but unknown tool. All sessions were audiotaped, and fidelity was assessed on an ongoing basis by a trained rater

Yes. To assess study fidelity, an MI specialist audited $10 \%$ of randomly selected phone calls and used MITI to code for the degree of empathy, direction, and MI techniques employed by the nurses

Yes. Fidelity to the intervention was maintained through videotaped review of $10 \%$ of each therapist's sessions, using the MITI scale

No. A clinical supervisor reviewed regularly the written material filled out by the nurses for each session. However, no objective fidelity assessment tool was used

No. The same nurse delivered all nursing interventions and, after each session, recorded if the pre-specified components of the adherence intervention had been delivered. No independent MI fidelity assessment mentioned

Yes, but unknown tool. Sample of calls were recorded, reviewed and graded by an MI expert 3 times during entire study period
MI nurses met every other week to discuss recent MI sessions and other relevant study issues. Periodic booster MI sessions were held

Counselors received ongoing weekly supervision of a random sample of selected audiotapes

No

MI trainer provided individualized feedback to the standardized patient interviews. No feedback mentioned for the live sessions

No

Not mentioned.

Not mentioned

Ongoing field monitoring throughout the study

An MI-trained clinical psychologist supervised and provided feedback on a biweekly basis

The rater provided feedback to the RAs based on the audiotape recordings

The MI specialist met once per month with the nursing supervisor and nurses to provide feedback on the calls and discuss strategies to improve further competencies and skills

Counselors received supervision throughout the project, but it was not mentioned if it was linked to the fidelity tool

Each month, each nurse had a supervision session with psychologists to discuss difficulties faced and potential solutions

A nurse experienced in HIV and substance abuse observed 3 sessions to give feedback on the pre-specified elements of the intervention but not on MI skills

The trainer gave structured feedback to the health educators using an assessment tool 
Table 3. (continued)

\begin{tabular}{llll}
\hline \hline Author & MI training & Fidelity assessment & Fidelity-based feedback \\
\hline Zwikker $^{31}$ & No details of training & $\begin{array}{l}\text { Yes, 4 of 9 pairs of sessions were } \\
\text { randomly audio-recorded. An } \\
\text { independent assessor used the BECCI } \\
\text { instrument to check MI 'ambiance' }\end{array}$ & Not mentioned \\
\hline
\end{tabular}

MITI: Motivational Interviewing Treatment Integrity

ART: Antiretroviral therapy

Stratifying the analyses according to the background of the interventionist revealed that MI improved adherence when delivered by nurses or research assistants with no specific degree. Interventions that used master level educators, psychologists or pharmacists did not show a significant MI effect. $^{17,22,24,25}$

Duration of MI exposure did not impact adherence. Using generalized least square regression, the exponentiated betacoefficient of change by tertiles of MI exposure was 0.90 (95\% CI 0.77-1.27; $\mathrm{p}=0.93$ ).

Among studies reporting an objective measure of adherence, the pooled RR of being adherent was 1.13 (95\% CI 1.01-1.28; $\mathrm{p}=0.03$ ) for the MI intervention when compared to the control group. The pooled RR of being adherent for MI compared to the control intervention was 1.15 (95\% CI 1.031.28; $\mathrm{p}=0.01$ ) among studies that used self-reported scales to ascertain adherence.

\section{DISCUSSION}

Our study reveals that MI improves medication adherence particularly among minority populations; however, the analysis of MI characteristics associated with this improvement had inconsistent results. This was due to the variability in adherence measures that required further stratification of the

Table 4 Point Estimates of the Different MI Characteristics on Medication Adherence

\begin{tabular}{|c|c|c|c|c|c|c|c|c|c|c|}
\hline \multicolumn{6}{|c|}{ Studies reporting medication adherence as a categorical variable } & \multicolumn{5}{|c|}{$\begin{array}{l}\text { Studies reporting medication adherence as a } \\
\text { continuous variable }\end{array}$} \\
\hline \multicolumn{11}{|c|}{ Effect of MI by delivery mode } \\
\hline Variable & $\mathrm{N}$ & $\mathrm{I}^{2}$ & RR & $95 \% \mathrm{CI}$ & p-value & $\mathrm{N}$ & $\mathrm{I}^{2}$ & SMD & $95 \% \mathrm{CI}$ & p-value \\
\hline Individual & 5 & 56 & 1.16 & $0.98-1.38$ & 0.07 & 5 & 93 & 0.80 & $-0.06-1.16$ & 0.06 \\
\hline Telephonic & 3 & 62 & 1.13 & $1.02-1.25$ & 0.02 & 3 & 93 & 0.92 & $-0.41-2.25$ & 0.17 \\
\hline Group & 3 & 98 & 1.55 & $0.54-4.44$ & 0.41 & 1 & $\mathrm{~N} / \mathrm{A}$ & 1.15 & $0.53-1.77$ & $<0.01$ \\
\hline Mixed & 0 & & & & & 3 & 79 & 0.25 & $-0.27-0.77$ & 0.35 \\
\hline \multicolumn{11}{|c|}{ Effect of MI by fidelity assessment } \\
\hline Fidelity tool & 7 & 87 & 1.17 & $0.99-1.36$ & 0.07 & 10 & 97 & 0.77 & $0.19-1.33$ & $<0.01$ \\
\hline No fidelity tool & 4 & 85 & 1.27 & $0.97-1.67$ & 0.08 & 1 & N/A & 0.00 & $-0.40-0.40$ & 1.0 \\
\hline \multicolumn{11}{|c|}{ Effect of fidelity-based feedback } \\
\hline MI feedback & 3 & 24 & 1.17 & $1.04-1.31$ & $<0.01$ & 5 & 98 & 0.60 & $-0.16-1.3$ & 0.12 \\
\hline No MI feedback & 8 & 88 & 1.16 & $1.00-1.31$ & 0.03 & 6 & 92 & 0.78 & $0.02-1.54$ & 0.04 \\
\hline \multicolumn{11}{|c|}{ Effect of MI by counselor background } \\
\hline Nurse & 4 & 71 & 1.32 & $1.04-1.68$ & 0.02 & 4 & 45 & 0.30 & $0.07-0.52$ & $<0.01$ \\
\hline Psychologist & 0 & & & & & 3 & 95 & 1.39 & $-0.49-3.20$ & 0.14 \\
\hline Master level & 2 & 85 & 1.37 & $0.55-3.39$ & 0.49 & 2 & 49 & -0.001 & $-0.26-0.26$ & 0.99 \\
\hline Pharmacist & 1 & $\mathrm{~N} / \mathrm{A}$ & 0.95 & $0.89-1.01$ & 0.13 & 0 & & & & \\
\hline Research Assistant & 4 & 40 & 1.12 & $1.02-1.23$ & 0.01 & 2 & 97 & 1.58 & $1.48-1.69$ & $<0.01$ \\
\hline \multicolumn{11}{|c|}{ Effect of MI by tertiles* of exposure time } \\
\hline Brief MI exposure & 3 & 0 & 1.23 & $1.08-1.50$ & $<0.01$ & 3 & 0 & 0.19 & $0.02-0.35$ & 0.02 \\
\hline Moderate MI exposure & 2 & 65 & 1.10 & $0.91-1.33$ & 0.34 & 3 & 79 & 0.28 & $0.05-0.50$ & 0.05 \\
\hline Longest MI exposure & 3 & 81 & 1.55 & $0.89-4.24$ & 0.09 & 3 & 94 & 1.28 & $0.88-1.6$ & $<0.01$ \\
\hline
\end{tabular}

$N$ : Number

$I^{2}:$ I squared

$R R$ : Relative risk

SMD: Standardized mean difference

N/A: Not applicable

MI: Motivational interviewing

*Description of tertiles: Categorical: tertile 1 (0-120), tertile 2 (121-480), tertile 3 (>481). Continuous: tertile 1 (0-90), tertile 2 (91-170), tertile 3 $(>170)$

Individual MI: categorical (22-24, 29, 30), continuous (17-19, 22, 29); telephonic studies: categorical (21, 28, 33), continuous (28, 33) (20); group MI: categorical (26, 32, 50), continuous (27); mixed MI: continuous (15, 25); fidelity assessment: categorical (22, 24, 27, 28, 30, 32), continuous (15, 15, 17-20, 22, 25, 27, 28, 33); no fidelity assessment: categorical (21, 23, 26, 29), continuous (29); fidelity-based feedback: categorical (28, 30, 33), continuous $(15,18,20,33,51) ;$ no fidelity-based feedback: categorical (21-24, 26, 27, 29, 32), continuous (19, 22, 25, 27, 29, 34)

Background of counselors: nurses: categorical (23, 26, 28, 29), continuous (15, 20, 28, 29); psychologist: continuous (17-19); pharmacists: categorical (32); master level: categorical $(22,24)$, continuous $(22,25)$; research assistant: categorical (16, 21, 30, 33) (21, 27, 30, 33) (21, 27, 30, 33)(21, 27, 30, 33) $(21,27,30,33)(22,38,40,43)$, continuous $(26,33)$

MI exposure time: tertile 1: categorical (28-30), continuous (18, 28, 29); tertile 2: categorical (23, 24), continuous (15, 20, 25); tertile 3: categorical (26, 27), continuous $(17,19,26)$ 
a

Study

ID

Telephonic

Palacio

Berger

Solomon

Subtotal $(1-$ squared $=62.3 \%, p=0.070)$

In-person individual

Ogedegbe

Samet

Pradier

Parsons

Golin

Subtotal $(I-$ squared $=56.6 \%, p=0.056)$

Group

Holstad

Holstad

Zwikker

Subtotal (I-squared $=97.5 \%, p=0.000)$

Overall (I-squared $=84.5 \%, p=0.000)$
$\operatorname{RR}(95 \% \mathrm{Cl}) \quad$ Weight

$1.28(1.09 .1 .50) \quad 11.11$

$1.08(1.03 .1 .14) \quad 14.11$

$1.10(0.97,1.25) \quad 12.24$

$1.13(1.02,1.25) \quad 37.46$

$1.32(0.96,1.81) \quad 6.74$

$1.05(0.78,1.40) \quad 7.34$

$1.21(1.00,1.47) \quad 10.25$

$1.03(0.91,1.16) \quad 12.36$

$2.01(1.02,3.98) \quad 2.29$

$1.16(0.98,1.38) \quad 38.99$

$3.02(1.68,5.42) \quad 2.94$

$1.36(0.99,1.87) \quad 6.75$

$0.95(0.89,1.02) \quad 13.86$

$1.55(0.54,4.44) \quad 23.55$

$1.17(1.05,1.31) \quad 100.00$

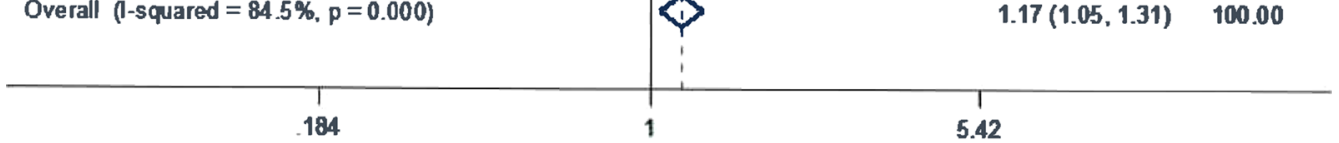

b

Study

ID

Telephonic

Palacio

Soloman

Subtotal (I-squared $=99.3 \%, p=0.000$ )

In-person individual

Golin

Ingersoll

Interian

Lavoie

Samet

Subtotal $(1-$ squared $=92.9 \%, p=0.000)$

Group

Holstad

Subtotal $(1$-squared $=\%, p=$.)

Mixed

Dilorio

Goggin

Konkle-Parker

Subtotal $(1$-squared $=79.5 \%, p=0.008$ )

Overall (I-squared $=97.0 \%, p=0.000$ )

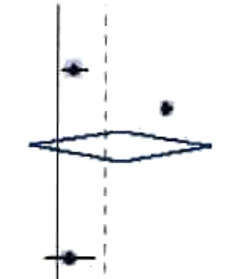

$\operatorname{SMD}(95 \% \mathrm{Cl}) \quad$ Weight

$0.24(0.05,0.43) \quad 9.69$

$1.60(1.50,1.70) \quad 9.77$

$0.92(-0.41,2.25) \quad 19.46$

$0.19(-0.18,0.55) \quad 9.40$

$0.34(-0.27,0.95) \quad 8.74$

$3.81(2.87,4.75) \quad 7.61$

$0.15(-0.39,0.68) \quad 8.97$

$0.00(-0.40,0.40) \quad 9.31$

$0.80(-0.06,1.66) \quad 44.03$

$1.15(0.53,1.77) \quad 8.72$

$1.15(0.53,1.77) \quad 8.72$

$0.59(0.26,0.91) \quad 9.48$

$-0.22(-0.62,0.17) \quad 9.33$

$0.37(-0.16,0.89) \quad 8.99$

$0.25(-0.27,0.77) \quad 27.80$

$0.70(0.15,1.25) \quad 100.00$

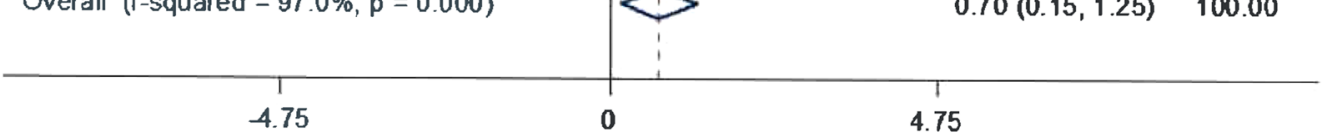

Figure 2 Effect of MI on adherence by the method of delivery of MI using (a) the proportion of subjects with adequate adherence (b) mean and standard deviation of the MI and control group 
analyses and the small samples of many studies that led to significant heterogeneity in effect size. MI characteristics associated with improved medication adherence were delivery by nurses or research assistants, telephonic MI and fidelitybased feedback among studies reporting categorical measures and group MI and fidelity assessment among studies reporting continuous measures.

Improving medication adherence is challenging given the complexity of its determinants and the heterogeneous adherence definitions. ${ }^{15,40,41}$ Minority and vulnerable populations are at an even higher risk of non-adherence. ${ }^{42-44}$

MI has been effective at improving medication adherence, ${ }^{5}$ however, the characteristics associated with efficacy have not been studied. ${ }^{33}$ This review suggests that primary care practices and community outreach clinics are effectively implementing and testing MI interventions. ${ }^{34}$ However, we found heterogeneity in delivery modes, number of training hours, fidelity assessment and provision of feedback, MI exposure time and outcome measurement.

When considering delivery modes, others had previously found phone-based MI to be promising, ${ }^{35}$ but the heterogeneity in quality and adherence measures limited their conclusions. Our meta-analyses found phone-based MI to be superior to usual care among RCTs only among studies reporting a categorical adherence measure, which had a more consistent effect size. The significantly larger effect size of Solomon et al., ${ }^{32}$ which reported a continuous measure, created heterogeneity that led to nonsignificant results. Similar limitations occurred in the in-person and group analyses in which Interian et al. ${ }^{19}$ and Holstad et al. ${ }^{26}$ also had larger effect sizes. It is important to clarify the efficacy of different MI delivery modes ${ }^{36}$ since they could improve MI's cost-effectiveness and reach among populations with access barriers. Solomon and Palacio et al. ${ }^{27}$, 32 used administrative pharmacy claims and a phone-based delivery mode to identify and reach a large number of eligible subjects over wide geographic areas. Group- or peer-led MI could offer a more comfortable environment for subjects reluctant to participate in one-on-one sessions. ${ }^{37}$

The evaluation of fidelity has been considered a marker of MI quality. ${ }^{5}$ Our study suggests that not evaluating MI fidelity is associated with a lack of impact on adherence. A limitation is that only a few studies did not report having fidelity assessment. Interestingly, even when most studies assessed fidelity, not many reported providing fidelity-based feedback, suggesting a need to evaluate in more depth the mechanisms by which fidelity improves the quality of MI.

As reported by others, ${ }^{3}$ level of education did not correlate with MI efficacy as the counselors associated with improved adherence had significantly different levels of education. This highlights the importance of MI training and the need to evaluate other innate counselors' characteristics that may be predictive of success. Similarly, total MI exposure time did not correlate with MI efficacy. However, the impact of MI frequency or overall program duration was not evaluated. More studies need to evaluate the sustainability and the long-term effect of MI.

Our study has several strengths. It furthers the knowledge that $\mathrm{MI}$ is effective at improving medication adherence ${ }^{3}$ by offering a rigorous evaluation of randomized control trials that focus exclusively on MI. Prior adherence studies have faced the challenge of short follow-up periods, low minority representation and lack of objective measures of adherence. ${ }^{38,39}$ The current review included studies with objective measures of adherence, large proportion of black subjects, wider range of follow-up and studies with repeated measures of adherence that allowed evaluating a possible dose effect. ${ }^{45}$

Our study has several limitations. First, most of the studies recruited black populations preferentially, and the percentage of studies focusing on non-minority subjects was low. The authors did not offer a rationale for the populations selected. Second, most of the interventions targeted antiretroviral medications limiting the generalizability of the results. However, most of the non-HIV studies showed MI as superior to the control group as well. Third, the power of our analyses was limited by the small sample sizes of many studies and the small number of studies in some of the subgroup analyses particularly when the between-study variance was large. Fourth, adherence measures were heterogeneous requiring stratification for analyses. Fifth, the recruitment of subjects with adequate baseline adherence could have impacted the effect size of MI and finally the internal validity of the studies could be threatened by quality issues such as large attrition rates, small sample sizes and missing data. However, most studies did report or conducted intention-to-treat analyses, used all data available and had balanced groups by completion status.

In summary, MI improves self-reported and objective measures of adherence to chronic medications after different lengths of exposure; however, the current evidence does not allow identifying consistent predictors of success. Larger studies are needed to confirm the efficacy of different delivery modes, fidelity assessment and feedback among diverse populations with a variety of chronic conditions. Efforts should be made to standardize the monitoring of adherence to chronic medications.

Corresponding Author: Ana Palacio, MD, MPH; University of Miami, $1120 \mathrm{NW}$ 14th St, Suite 1144, Miami, FL 33136, USA (e-mail: apalacio2@med.miami.edu).

\section{Compliance with Ethical Standards:}

Conflict of Interest: The authors declare that they do not have a conflict of interest.

\section{REFERENCES}

1. Osterberg L, Blaschke T. Adherence to medication. N Engl J Med. 2005;353(5):487-97.

2. Ho PM, Rumsfeld JS, Masoudi FA, et al. Effect of medication nonadherence on hospitalization and mortality among patients with diabetes mellitus. Arch Intern Med. 2006;166(17):1836-41. 
3. Easthall C, Song F, Bhattacharya D. A meta-analysis of cognitive-based behaviour change techniques as interventions to improve medication adherence. BMJ Open. 2013;3(8):10.1136/bmjopen,2013-002749.

4. Lundahl B, Moleni T, Burke BL, et al. Motivational interviewing in medical care settings: a systematic review and meta-analysis of randomized controlled trials. Patient Educ Couns. 2013;93(2):157-68.

5. Miller WR, Rollnick S. Meeting in the middle: motivational interviewing and self-determination theory. Int $\mathrm{J}$ Behav Nutr Phys Act. 2012;9:25. doi:10.1186/1479-5868-9-25.

6. Thompson DR. Motivational interviewing improves patients' mood and reduces mortality 12 months poststroke. Evid Based Nurs. 2011;15(2):35. doi:10.1136/ebn.2011.100200.

7. Tripp SB, Perry JT, Romney S, Blood-Siegfried J. Providers as weight coaches: using practice guides and motivational interview to treat obesity in the pediatric office. J Pediatr Nurs. 2011;26(5):474-9.

8. Carroll KM, Ball SA, Nich C, et al. Motivational interviewing to improve treatment engagement and outcome in individuals seeking treatment for substance abuse: a multisite effectiveness study. Drug Alcohol Depend. 2006;81(3):301-12.

9. Steiner JF, Prochazka AV. The assessment of refill compliance using pharmacy records: methods, validity, and applications. J Clin Epidemiol. 1997;50(1):105-16.

10. Madson MB, Loignon AC, Lane C. Training in motivational interviewing: a systematic review. J Subst Abus Treat. 2009;36(1):101-9.

11. Greenland S, Longnecker MP. Methods for trend estimation from summarized dose-response data, with applications to meta-analysis. Am J Epidemiol. 1992;135(11):1301-9.

12. Moher D, Liberati A, Tetzlaff J, Altman DG, PRISMA Group. Preferred reporting items for systematic reviews and meta-analyses: the PRISMA statement. Ann Intern Med. 2009; 151(4):264-9. W64.

13. Sterne JA, Gavaghan D, Egger M. Publication and related bias in metaanalysis: power of statistical tests and prevalence in the literature. J Clin Epidemiol. 2000;53(11):1119-29.

14. Berkey CS, Hoaglin DC, Mosteller F, Colditz GA. A random-effects regression model for meta-analysis. Stat Med. 1995;14(4):395-411.

15. Konkle-Parker DJ, Erlen JA, Dubbert PM, May W. Pilot testing of an HIV medication adherence intervention in a public clinic in the deep south. $\mathrm{J}$ Am Acad Nurse Pract. 2012;24(8):488-98.

16. Holstad MM, Essien JE, Ekong E, Higgins M, Teplinskiy I, Adewuyi MF. Motivational groups support adherence to antiretroviral therapy and use of risk reduction behaviors in HIV positive Nigerian women: a pilot study. Afr J Reprod Health. 2012;16(3):14-27.

17. Ingersoll KS, Farrell-Carnahan L, Cohen-Filipic J, et al. A pilot randomized clinical trial of two medication adherence and drug use interventions for HIV+ crack cocaine users. Drug Alcohol Depend. 2011;116(1-3):177-87.

18. Lavoie KL, Moullec G, Lemiere C, et al. Efficacy of brief motivational interviewing to improve adherence to inhaled corticosteroids among adult asthmatics: results from a randomized controlled pilot feasibility trial. Patient Prefer Adherence. 2014;8:1555-69.

19. Interian A, Lewis-Fernandez R, Gara MA, Escobar JI. A randomizedcontrolled trial of an intervention to improve antidepressant adherence among Latinos with depression. Depress Anxiety. 2013;30(7):688-96.

20. DiIorio C, McCarty F, Resnicow $\mathbf{K}$, et al. Using motivational interviewing to promote adherence to antiretroviral medications: a randomized controlled study. AIDS Care. 2008;20(3):273-83.

21. Berger BA, Liang $\mathbf{H}$, Hudmon KS. Evaluation of software-based telephone counseling to enhance medication persistency among patients with multiple sclerosis. J Am Pharm Assoc (2003). 2005;45(4):466-72.

22. Golin CE, Earp J, Tien HC, Stewart P, Porter C, Howie L. A 2-arm, randomized, controlled trial of a motivational interviewing-based intervention to improve adherence to antiretroviral therapy (ART) among patients failing or initiating ART. J Acquir Immune Defic Syndr. 2006;42(1):42-51.

23. Pradier C, Bentz L, Spire B, et al. Efficacy of an educational and counseling intervention on adherence to highly active antiretroviral therapy: French prospective controlled study. HIV Clin Trials. 2003;4(2):121-31

24. Parsons JT, Golub SA, Rosof E, Holder C. Motivational interviewing and cognitive-behavioral intervention to improve HIV medication adherence among hazardous drinkers: a randomized controlled trial. J Acquir Immune Defic Syndr. 2007;46(4):443-50.

25. Goggin K, Gerkovich MM, Williams KB, et al. A randomized controlled trial examining the efficacy of motivational counseling with observed therapy for antiretroviral therapy adherence. AIDS Behav. 2013;17(6):1992-2001

26. Holstad MM, Dilorio C, Kelley ME, Resnicow K, Sharma S. Group motivational interviewing to promote adherence to antiretroviral medications and risk reduction behaviors in HIV infected women. AIDS Behav. 2011;15(5):885-96.

27. Palacio AM, Uribe C, Hazel-Fernandez L, et al. Can phone-based motivational interviewing improve medication adherence to antiplatelet medications after a coronary stent among racial minorities? A randomized trial. J Gen Intern Med. 2015;30(4):469-75. doi:10.1007/s11606-0143139-8.

28. Samet JH, Horton NJ, Meli S, et al. A randomized controlled trial to enhance antiretroviral therapy adherence in patients with a history of alcohol problems. Antivir Ther. 2005;10(1):83-93.

29. Ogedegbe G, Chaplin W, Schoenthaler A, et al. A practice-based trial of motivational interviewing and adherence in hypertensive African Americans. Am J Hypertens. 2008;21(10):1137-43.

30. Dilorio C, Resnicow K, McDonnell M, Soet J, McCarty F, Yeager K. Using motivational interviewing to promote adherence to antiretroviral medications: a pilot study. J Assoc Nurses AIDS Care. 2003;14(2):52-62.

31. Zwikker HE, van den Ende $\mathbf{C H}$, van Lankveld WG, et al. Effectiveness of a group-based intervention to change medication beliefs and improve medication adherence in patients with rheumatoid arthritis: a randomized controlled trial. Patient Educ Couns. 2014;94(3):356-61.

32. Solomon DH, Iversen MD, Avorn J, et al. Osteoporosis telephonic intervention to improve medication regimen adherence: a large, pragmatic, randomized controlled trial. Arch Intern Med. 2012;172(6):477-83.

33. Longabaugh $\mathbf{R}$. Comments on dunn et al.'s "the use of brief interventions adapted from motivational interviews across behavioral domains: a systematic review". Why is motivational interviewing effective? Addiction. 2001;96(12): 1773-4. discussion 1774-5.

34. Bellg AJ, Borrelli B, Resnick B, et al. Enhancing treatment fidelity in health behavior change studies: best practices and recommendations from the NIH behavior change consortium. Health Psychol. 2004;23(5):443-51.

35. Teeter BS, Kavookjian J. Telephone-based motivational interviewing for medication adherence: a systematic review. Transl Behav Med. 2014;4(4):372-81.

36. Cutler DM, Everett w. Thinking outside the pillbox-medication adherence as a priority for health care reform. N Engl J Med. 2010;362(17):1553-5.

37. Broadhead RS, Heckathorn DD, Weakliem DL, et al. Harnessing peer networks as an instrument for AIDS prevention: results from a peer-driven intervention. Public Health Rep. 1998;113(Suppl 1):42-57.

38. Schroeder K, Fahey T, Ebrahim S. Interventions for improving adherence to treatment in patients with high blood pressure in ambulatory settings. Cochrane Database Syst Rev. 2004;(2):CD004804.

39. McDonald HP, Garg AX, Haynes RB. Interventions to enhance patient adherence to medication prescriptions: scientific review. JAMA. 2002;288(22):2868-79.

40. Gellad WF, Grenard JL, Marcum ZA. A systematic review of barriers to medication adherence in the elderly: looking beyond cost and regimen complexity. Am J Geriatr Pharmacother. 2011;9(1):11-23.

41. Ickovics JR, Meisler AW. Adherence in AIDS clinical trials: a framework for clinical research and clinical care. J Clin Epidemiol. 1997;50(4):385-91.

42. Chowdhury R, Khan H, Heydon $\mathbf{E}$, et al. Adherence to cardiovascular therapy: a meta-analysis of prevalence and clinical consequences. Eur Heart J. 2013;34(38):2940-8.

43. Kumbhani DJ, Steg PG, Cannon CP, et al. Adherence to secondary prevention medications and four-year outcomes in outpatients with atherosclerosis. Am J Med. 2013;126(8):693-700. e1.

44. Lewey J, Shrank WH, Bowry AD, Kilabuk E, Brennan TA, Choudhry NK. Gender and racial disparities in adherence to statin therapy: a metaanalysis. Am Heart J. 2013;165(5):665-78. 678.e1.

45. Chung MH, Richardson BA, Tapia K, et al. A randomized controlled trial comparing the effects of counseling and alarm device on HAART adherence and virologic outcomes. PLoS Med. 2011;8(3):e1000422. 